\title{
Prospecção científica e tecnológica do uso de nanopartículas com alendronato de sódio no tratamento de câncer
}

Scientific and technological prospection of the use of nanoparticles with sodium alendronate in cancer treatment

Prospección científica y tecnológica del uso de nanopartículas con alendronato de sodio en el tratamiento del cáncer

Recebido: 11/09/2021 | Revisado: 19/09/2021 | Aceito: 20/09/2021 | Publicado: 21/09/2021

Fabrício dos Santos Machado ORCID: https://orcid.org/0000-0003-1637-5934 Universidade Federal do Delta do Parnaíba, Brasil E-mail: fabricio.machado10@live.com

Bruno Iles

ORCID: https://orcid.org/0000-0001-6844-7608 Universidade Federal do Delta do Parnaíba, Brasil E-mail: iles630@gmail.com

Rodrigo Elísio de Sá

ORCID: https://orcid.org/0000-0002-1492-3995

Universidade Federal do Delta do Parnaíba, Brasil E-mail: rodrigosa238@gmail.com

Dakson Douglas Araújo

ORCID: https://orcid.org/0000-0003-0102-7240 Universidade Federal do Delta do Parnaíba, Brasil E-mail: dacksondouglas@gmail.com

Gabriella Linhares de Andrade ORCID: https://orcid.org/0000-0003-2481-9646 Universidade Federal do Delta do Parnaíba, Brasil E-mail: gabriellalinhares9@gmail.com

Adryele Ribeiro Arantes

ORCID: https://orcid.org/0000-0003-4750-6466

Universidade Federal do Delta do Parnaíba, Brasil E-mail: adryeler@gmail.com Gabrielle Costa Sousa

ORCID: https://orcid.org/0000-0001-8496-3477 Universidade Federal do Delta do Parnaíba, Brasil E-mail: gabygabryelle19@hotmail.com

Lucicleia Dias Monteiro

ORCID: https://orcid.org/0000-0002-6904-4787 Universidade Federal do Delta do Parnaíba, Brasil E-mail: Lucicleiabiomed@gmail.com

Antonio Carlos Pereira de Oliveira ORCID: https://orcid.org/0000-0003-0136-5944 Universidade Federal do Delta do Parnaíba, Brasil E-mail: antoniocarlosbio@outlook.com

Luma Brisa Pereira dos Santos ORCID: https://orcid.org/0000-0003-1912-6554 Universidade Federal do Delta do Parnaíba, Brasil E-mail: lumabrisa3@gmail.com

Maria Syndel Caroline Ribeiro Franco ORCID: https://orcid.org/0000-0002-7765-3347

Universidade Federal do Delta do Parnaíba, Brasil E-mail: syndelcaroline@ hotmail.com 


\begin{abstract}
Resumo
Câncer é o nome dado a um conjunto de doenças complexas caracterizadas pelo crescimento descontrolado de células anormais com alto potencial invasivo, sendo considerado um dos maiores problema de saúde pública mundial. A incidência de câncer tem aumentado a cada ano, mostrando a relevância na realização de pesquisas cujo objetivo seja o tratamento do câncer em suas diversas modalidades. Assim, este trabalho tem como objetivo realizar uma prospecção científica e tecnológica sobre a aplicação de nanopartículas com alendronato de sódio no tratamento de câncer. Para isso, utilizou-se as seguintes palavras-chave, com o aperador booleano AND, nas buscas: "Sodium alendronate and nanoparticle", "Sodium alendronate and cancer", "Nanoparticle and cancer". Os resultados demonstraram na prospecção científica uma grande quantidade de trabalhos relacionados com o câncer quando associado com nanopartículas. Constatou-se que nos últimos 10 anos houve um crescimento linear na produção científica voltada para esse tema. O mesmo também foi observado na busca por patentes, onde foram observados até 37.100 pedidos de patente com esses termos em sua estrutura, na base Espace Net. Esses dados apontam a preocupação da comunidade científica com a melhoria no tratamento da neoplasia, e evidenciam a associação com a nanotecnologia uma alternativa viável e promissora.
\end{abstract}

Palavras-chave: Nanopartículas; Alendronado de sódio; Câncer.

\begin{abstract}
Cancer is the name given to a set of complex diseases characterized by the uncontrolled growth of abnormal cells with a high invasive potential, being considered one of the biggest public health problems in the world. The incidence of cancer has increased every year, showing the relevance of conducting research aimed at treating cancer in its various modalities. Thus, this work aims to carry out a scientific and technological prospection on the application of nanoparticles with sodium alendronate in the treatment of cancer. For this, the following keywords were used, with the Boolean operator AND, in the searches: "Sodium alendronate and nanoparticle", "Sodium alendronate and cancer", "Nanoparticle and cancer". The results demonstrated in scientific prospecting a large amount of work related to cancer when associated with nanoparticles. It was found that in the last 10 years there has been a linear growth in scientific production focused on this topic. The same was observed in the search for patents, where up to 37,100 patent applications were observed with these terms in their structure, in the Espace Net database. with nanotechnology a viable and promising alternative.
\end{abstract}

Keywords: Nanopartículas; Alendronato de sodio; Cancer.

\title{
Resumen
}

Cáncer es el nombre que se le da a un conjunto de enfermedades complejas caracterizadas por el crecimiento descontrolado de células anormales con un alto potencial invasivo, siendo considerado uno de los mayores problemas de salud pública del mundo. La incidencia de cáncer ha aumentado cada año, lo que demuestra la relevancia de realizar investigaciones dirigidas al tratamiento del cáncer en sus diversas modalidades. Así, este trabajo tiene como objetivo realizar una prospección científica y tecnológica sobre la aplicación de nanopartículas con alendronato de sodio en el tratamiento del cáncer. Para ello, se utilizaron las siguientes palabras clave, con el operador booleano AND, en las búsquedas: "Alendronato de sodio y nanopartícula", "Alendronato de sodio y cáncer", "Nanopartícula y cáncer". Los resultados demostraron en la prospección científica una gran cantidad de trabajos relacionados con el cáncer cuando se asocia con nanopartículas. Se encontró que en los últimos 10 años ha habido un crecimiento lineal en la producción científica enfocada en este tema. Lo mismo se observó en la búsqueda de patentes, donde se observaron hasta 37.100 solicitudes de patentes con estos términos en su estructura, en la base de datos Espace Net. Con la nanotecnología como alternativa viable y prometedora.

Palabras clave: Nanopartículas; Alendronato de sodio; Cáncer.

\section{Introdução}

O câncer é considerado uma doença complexa e de difícil tratamento, caracterizado por uma série de doenças de fundo genético e/ou epigenético, que envolve crescimento anormal de células com potencial invasivo, e é uma doença de pesquisa para o desenvolvimento ou descoberta de novos fármacos (Andreopoulou; Kelly; Mcdaid, 2017; Steenbruggen et al, 2017). No Brasil, as taxas de mortalidades são elevadas devido ao diagnóstico tardio e a complicações dos estágios avançados da doença (Abbas; Rehman, 2018).

Para isto, a busca por novos tratamentos têm sido alvos de pesquisas com o intuito de encontrar um composto eficiente contra linhagens cancerosas, além de possivelmente impedir o desenvolvimento de processos de metastáticos. Entre estes, encontram-se os compostos bifosfonatos (BPs), como o alendronato de sódio. Estes compostos foram descritos como 
eficientes no tratamento de osteopenias e osteoporoses, além de ajudar a impedir metástases ósseas. De acordo com a literatura é mostrado também sua atividade contra linhagens de câncer de mama com receptor de estrogênio positivo e negativo (Oliveira et al; 2011).

BPs são análogos estáveis do pirofosfato inorgânico e possuem alta afinidade pela matriz óssea mineral, onde se ligam fortemente à hidroxiapatita da superfície óssea, especialmente em locais onde há intensa remodelação tecidual (Russel; Rogers, 1999). Clinicamente os BPs são usados no combate à inibição da reabsorção óssea, sendo tratamento de escolha para uma variedade de patologias onde há atividade osteoclástica excessiva, tais como doença óssea de Paget, osteoporose e doença óssea metastática osteolítica (Van den Wyngaert et al., 2009). Atualmente estão bem estabelecidos os efeitos benéficos dos BPs em complicações ósseas associadas a malignidades, como hipercalcemia, dor, destruição óssea e fraturas patológicas, possibilitando aumento da sobrevida e redução da morbidade dos pacientes (Coleman; Mccloskey, 2011; Russell, 2011).

Os BPs não são utilizados apenas para tratamento e prevenção da osteopenia e osteoporose, mas também na redução de fraturas associadas a metástases ósseas. Ademais, estudos em usuárias de BPs orais e endovenosos também revelaram a possibilidade de redução à recorrência locorregional de tumores de mama. Chlebowski et al. (2010), da Universidade de Winsconsin, verificaram por meio de um estudo caso-controle com seguimento de três anos, com 2.936 pacientes com câncer de mama e 2.975 pacientes sem a doença, que o uso de BPs mostrou-se fator redutor de risco da doença nos pacientes.

Alguns estudos constataram redução de 35\% na recorrência da doença nas portadoras de câncer de mama após a menopausa que medicadas com BPs a cada seis meses, enquanto no estudo AZURE (Adjuvant Zoledronic acid to redUce Recurrence) o uso de BPs na neoadjuvância dobrou a taxa de remissão completa da doença (Neville-Webbe et al., 2010). Esses resultados sugerem que o mecanismo envolvido pode ser inibitório direto contra o câncer de mama.

Os mecanismos pelos quais os BPs podem reduzir a incidência do câncer de mama ainda não estão definidos. Postulase, com base em estudos pré-clínicos, que esse fármaco pode atuar sobre a angiogênese por meio da diminuição de Fator de Crescimento Endotelial Vascular (VEGF), induzindo a apoptose, além de reduzir a proliferação e impedir a invasão da matriz extracelular (Lipton, 2008). Esses mecanismos diferem daqueles envolvidos na ação dos moduladores seletivos de receptor de estrogênio (SERMs), nos quais a proteção ocorre exclusivamente nos cânceres de mama ER-positivos (Cataliotti, et al., 2006) enquanto que, com os BPs, a proteção estende-se aos casos de cânceres ER-negativos, que correspondem a 30\% dos cânceres mamários. Apesar desses dados justificarem uma possível ação quimiopreventiva dos dessa classe dos BPs em relação ao câncer de mama, ainda é muito prematuro disponibilizá-lo para sua prevenção e, por isso, são necessários estudos randomizados para testar sua real eficácia para uso clínico (Oliveira; Aldrighi; Bagnoli, 2011).

O Bifosfonato Alendronato de Sódio (ALD) é descrito na literatura como uma droga tóxica e corrosiva para mucosas gástricas, agindo de forma local no estômago. Devido a isso, este composto foi demonstrado com uso limitado por causar desconforto gástrico como dor abdominal, diarreia e ulcerações envolvendo o esôfago e o estômago (Papamitsou et al., 2019). Desse modo, uma terapia eficaz para o tratamento do câncer de mama, minimizando as lesões gástricas e outros efeitos colaterais, induzidas por alendronato poderia ser estabelecido investigando os mecanismos que contribuem para o ferimento da mucosa gástrica, buscando moléculas sinalizadoras que amplificariam o mecanismo de defesa da mucosa ou associar o ALD com drogas ou moléculas gastroprotetoras.

Assim, as nanopartículas constituídas por polímeros naturais biodegradáveis têm mostrado grande potencial como veículos para transporte de fármacos, atraindo muito a atenção dos pesquisadores devido às suas potencialidades terapêuticas, tais como: manutenção do nível terapêutico do fármaco no sangue, diminuição das reações adversas e melhora do esquema posológico através da diminuição do número de doses (Schaffazick et al., 2013).

A nanotecnologia tem avançado rapidamente nos últimos anos, encontrando aplicações nas mais diversas áreas, desde 
setores de energia e eletrônica até a indústria farmacêutica (Ye et al., 2018). Um dos setores nanotecnológicos com maior potencial de aplicação são os sistemas de carregamento e liberação de drogas, para melhorar a sua eficácia terapêutica (Mniqbal et al., 2016). Nanopartículas poliméricas podem apresentar-se na forma de nanocápsulas ou nanoesferas, respectivamente. As nanocápsulas são constituídas por um invólucro polimérico disposto ao redor de um núcleo oleoso, podendo o fármaco estar dissolvido neste núcleo e/ou adsorvido à parede polimérica. E as nanoesferas não contêm óleo na sua formulação, mas são formadas por uma matriz polimérica, onde o fármaco pode ficar retido ou adsorvido (Schaffazick et al., 2013).

Os polissacarídeos apresentam características que podem ser adequadas a nanopartículas e aplicáveis na área médica. Características como: baixo custo, disponibilidade, biocompatível e características biodegradáveis. O seu reconhecimento molecular é uma das principais vantagens da utilização de polissacarídeos como componentes para a síntese de nanopartículas, uma vez que eles têm receptores específicos em certas células (Iles et al., 2021).

Nesse sentido, o mapeamento científico e tecnológico de pesquisas em relação a propriedades farmacológicas anticâncer de nanopartículas poliméricas com alendronato de sódio tornam-se relevante para a identificação das aplicações científicas e tecnológicas. Assim sendo, prospecções permitem mapear sistematicamente estudos no intuito de propor uma aplicação inovadora em prol de amparar as pesquisas nos moldes da farmacologia (Sousa et al., 2021).

Diante desse contexto, a utilização de nanopartículas poliméricas como um novo veículo de liberação de alendronato de sódio demonstra grande importância, visto a possibilidade de aplicação de um novo produto com maior eficácia e biodisponibilidade, bem como menores efeitos colaterais no tratamento do câncer de mama.

\section{Metodologia}

Pesquisa e mapeamento de informações é uma ferramenta importante para reunir dados relevantes na literatura e consequentemente abrir perspectivas para novos estudos (Soares et al., 2020). Para o desenvolvimento desta prospecção científica e tecnológica, foi mapeado informações acerca de nanopartículas com alendronato de sódio associado ao câncer. Dessa forma, foram realizadas buscas em bancos de dados nacionais e internacionais de artigos científicos e patentes, tais como: Instituto Nacional da Propriedade Industrial (INPI), Europe's Network of Patents databases (Espace net), United States Patent and Trademark Office (USPTO) e Thompson Reuters (Web of Science), e as bases utilizadas para a pesquisa de artigos foram: Chemical Society (ACS), Portal Regional da Biblioteca Virtual em Saúde (Bireme), Google acadêmico, Nacional Center for Biotechnology information (PubMed), Scientífic Eletronic Library Online (SciELO), Science Direct, grupo Elsevier Group (Scopus) e Thompson Reuters (Web of Science). As bases aqui utilizadas são exemplificadas na Figura 1.

Para tanto, considerou-se os documentos em inglês e português que apresentavam as palavras-chave no título e/ou no resumo, sendo os descritores utilizados para a busca: "Sodium alendronate and nanoparticle", "Sodium alendronate and cancer", "Nanoparticle and cancer". Utilizaram-se todos os trabalhos relacionados e relavantes que serviriam de base para a realização e montagem da prospecção. Os dados prospectados foram obtidos de novembro à dezembro de 2020 . Além das palavras-chave, a pesquisa foi realizada com a utilização de alguns recursos, como as aspas, para ajudar a obter o maior número possível de documentos juntamente com o operador booleano AND entre as palavras. Depois dos resultados obtidos, foi realizada uma comparação entre os artigos a fim de se avaliar o andamento das pesquisas sobre o uso dos bifosfonados no tratamento do câncer, bem como o uso da nanotecnologia nesse processo. Portanto, estudos desse cunho são importantes pois abrem oportunidades para novas pesquisas científicas e contribui para o desenvolvimento biotecnológico (Sousa et al., 2021). 
Figura 1 - Organograma das bases de dados utilizadas para o desenvolvimento da prospecção científica e tecnológica acercado uso de nanopartículas poliméricas com alendronato de sódio associado ao câncer.

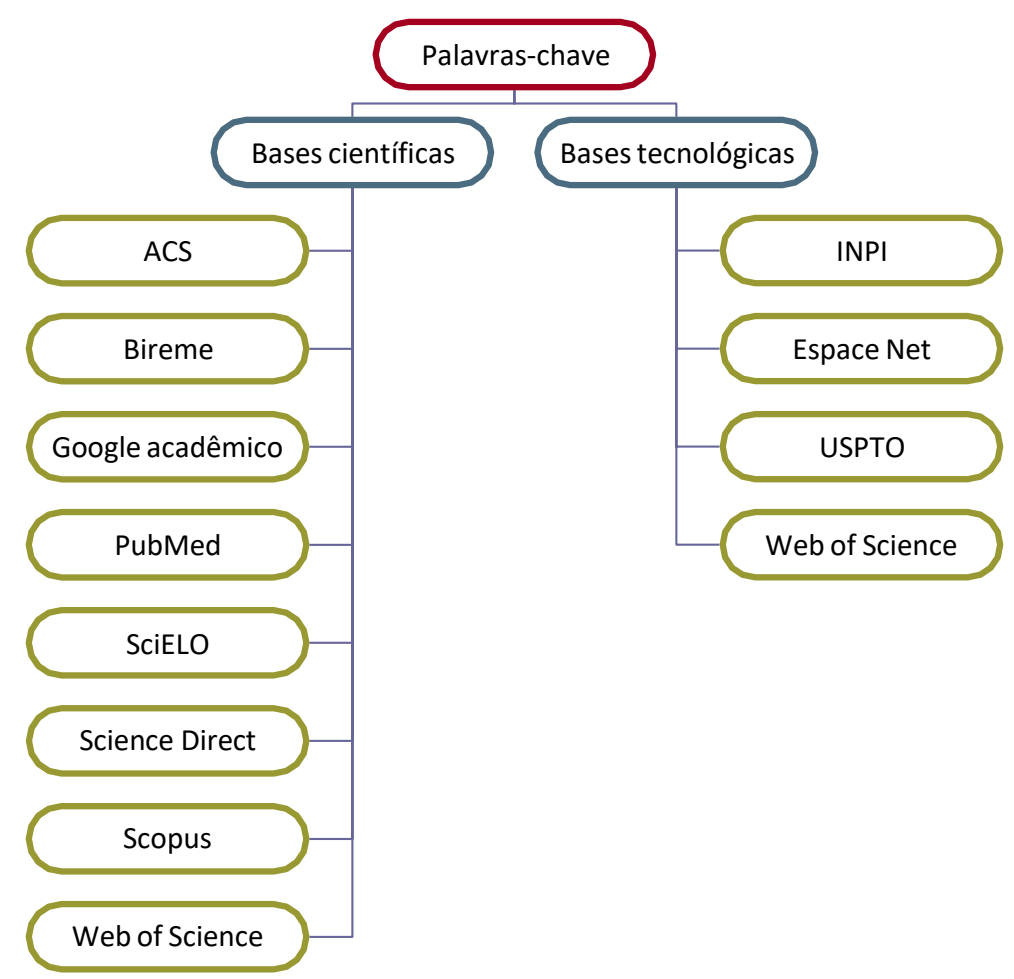

Fonte: Autores (2021).

\section{Resultados e Discussão}

De acordo com as últimas estimativas do Instituto Nacional do Câncer para o biênio 2020-2022, estima-se que haja 625.000 novos casos de câncer a cada ano, dos quais aproximadamente 177.000 são de pele não melanoma (Figura 2). No Brasil, a incidência estimada de cânceres relacionados ao gênero difere do resto do mundo, indicando que o câncer de próstata (66.000) em homens e o câncer de mama (66.000) em mulheres são os mais comuns (INCA, 2019). Como resultado, a OMS incentiva os países a priorizarem serviços básicos de diagnóstico e tratamento de alto impacto e baixo custo, pois a detecção precoce do câncer pode reduzir o impacto final da doença, além de oferecer tratamentos mais eficazes, simples e baratos, que proporcionam maiores chances de cura.

Figura 2 - Distribuição proporcional dos dez tipos de câncer mais incidentes estimados para 2022 por sexo, exceto pele não melanoma*.

\begin{tabular}{|c|c|c|c|c|c|c|c|}
\hline Localizaçáo Primária & Casos & $\%$ & & & Localizaçáo Primária & Casos & $\%$ \\
\hline Próstata & 65.840 & $29,2 \%$ & Homens & Mulheres & Mama feminina & 66.280 & $29,7 \%$ \\
\hline Colon e reto & 20.520 & $9,1 \%$ & & & Cólon e reto & 20.470 & $9,2 \%$ \\
\hline Traqueia, brónquio e pulmáo & 17.760 & $7,9 \%$ & & & Colo do útero & 16.590 & $7.4 \%$ \\
\hline Estómago & 13.360 & $5,9 \%$ & & & Traqueia,brónquio e pulmâo & 12.440 & $5,6 \%$ \\
\hline Cavidade oral & 11,180 & $5,0 \%$ & & & Glāndula tireoide & 11.950 & $5,4 \%$ \\
\hline Esôfago & 8.690 & $3,9 \%$ & & & Estômago & 7.870 & $3,5 \%$ \\
\hline Bexiga & 7.590 & $3,4 \%$ & & & Ovário & 6.650 & $3,0 \%$ \\
\hline Linfoma não Hodgkin & 6.580 & $2,9 \%$ & & & Corpo do úfero & 6.540 & $2,9 \%$ \\
\hline Laringe & 6.470 & $2,9 \%$ & & & Linfoma não Hodgkin & 5.450 & $2,4 \%$ \\
\hline Leucemias & 5.920 & $2,6 \%$ & & & Sistema nervoso central & 5.220 & $2,3 \%$ \\
\hline
\end{tabular}

*Números arredondados para múltiplos de 10.

Fonte: INCA (2019). 
Desta forma, a ciência está sempre em busca de novas estratégias para combater a progressão de tumores cancerosos e melhorar o tratamento para os pacientes. Porém, os resultados nem sempre são satisfatórios, pois, além de causar diversos efeitos colaterais, essas novas estratégias apresentam alto índice de recorrência tumoral e aparecimento de metástases. Portanto, a pesquisa baseada em terapia direcionada cresceu significativamente. A pesquisa sobre as diferentes características das células cancerosas levou ao desenvolvimento de drogas direcionadas, que são compostos que atuam nas células tumorais com base nas características que as tornam diferentes das células normais e das células saudáveis, reduzindo assim os efeitos colaterais (Baudino, 2015).

Foi possível verificar na prospecção científica uma grande quantidade de trabalhos relacionados com o câncer quando associado com nanopartículas, com 92.000 trabalhos encontrados no Google acadêmico e 54.167 na base PubMed (Tabela 1 e Figura 3). Isso mostra que essa busca por novos tratamentos está em crescimento e que a nanotecnologia está se mostrando um ramo promissor nessa área. Esses dados corroboram com o delineamento temporal (Figigura 4), onde é possível observar que nos últimos 10 anos houve um crescimento linear na produção científica voltada para esse tema. O mesmo foi observado na busca por patentes, onde foram observados até 37.100 pedidos de patente com esses termos em sua estrutura, na base Espace Net, esses dados juntos apontam a preocupação da comunidade científica com a melhoria no tratamento da neoplasia, e evidenciama associação com a nanotecnologia uma alternativa viável e promissora (Figura 5).

Figura 3 - Depósito de artigos relacionados a alendronato de sódio, nanopartículas e câncer nas principais plataformas de pesquisa científica.

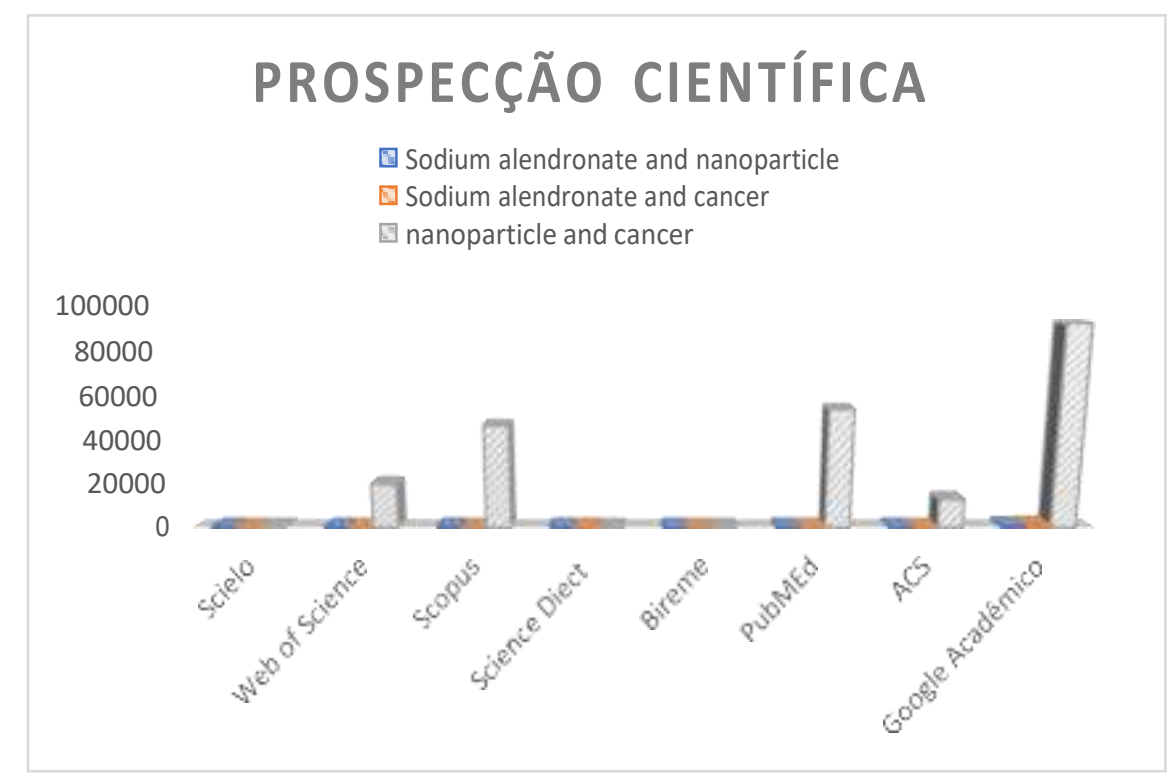

Fonte: Autores (2021).

A nanotecnologia possui um conceito que foi aprimorado com o desenvolvimento da ciência, portanto ainda não há um consenso internacional sobre o termo. Desta forma, a Agência Europeia de Medicamentos define nanopartículas como estruturas com propriedades específicas com um diâmetro inferior a 1.000 nanômetros. O princípio dessa nova ciência é que materiais em nanoescala podem ter diferentes propriedades químicas, físico-químicas e comportamentais em comparação com materiais apresentados em grande escala (Dimer et al., 2013). De acordo com suas capacidades de biodegradabilidade, biocompatibilidade, funcionalização de superfície, ligação, complexação e encapsulação, são selecionados os materiais utilizados para a obtenção de preparações nanotecnológicas. (Idrees et al., 2020). 
Figura 4 - Aspecto temporal do depósito de artigos relacionados a alendronato de sódio, nanopartículas e câncer nas principais plataformas de pesquisa científica.

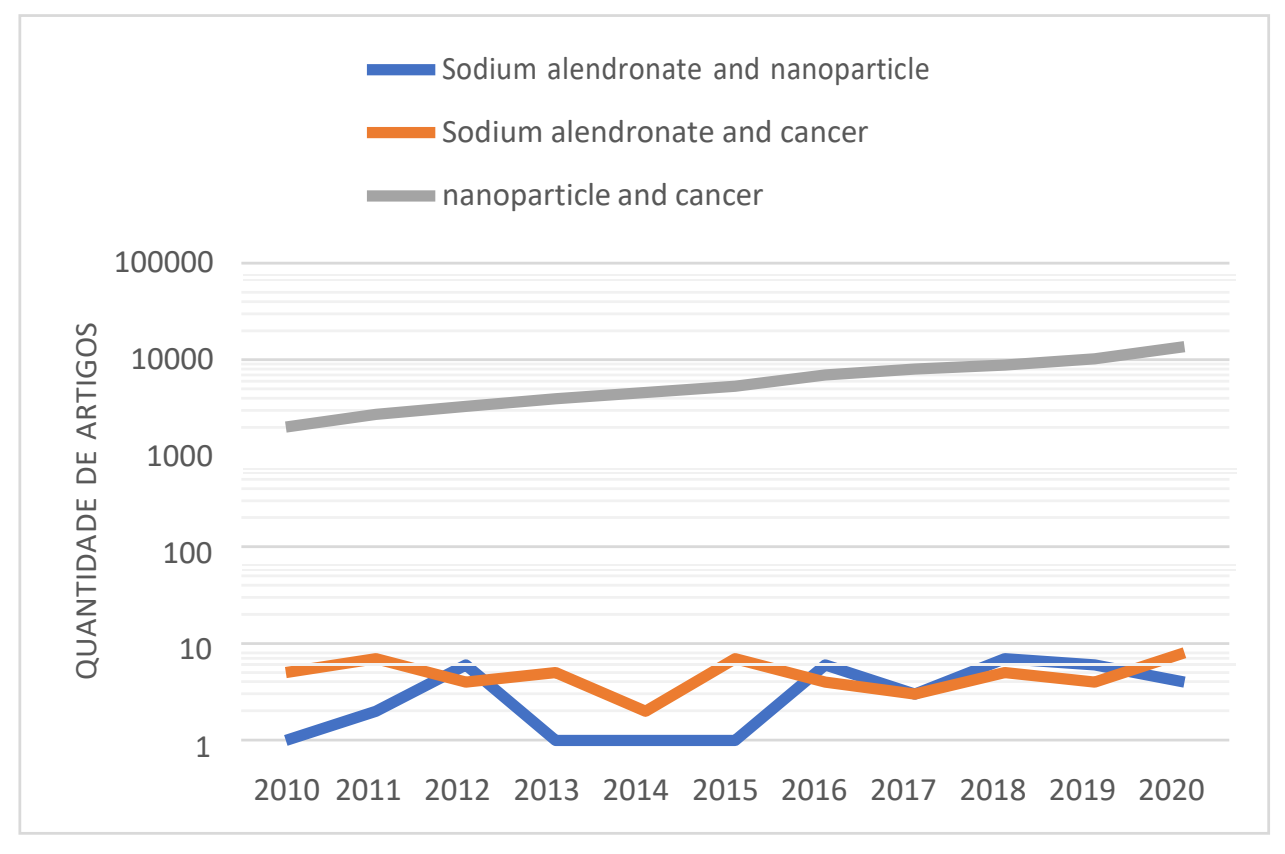

Fonte: Autores (2021).

Tabela 1 - Quantificação de artigos encontrados nas bases de pesquisa científica.

\begin{tabular}{|c|c|c|c|c|c|c|c|c|}
\hline Palavras-chave & Scielo & $\begin{array}{l}\text { Web of } \\
\text { Science }\end{array}$ & Scopus & $\begin{array}{l}\text { Science } \\
\text { Diect }\end{array}$ & Bireme & PubMed & ACS & $\begin{array}{c}\text { Google } \\
\text { Acadêmico }\end{array}$ \\
\hline $\begin{array}{l}\text { Sodium alendronate } \\
\text { and nanoparticle }\end{array}$ & 0 & 4 & 8 & 34 & 0 & 26 & 5 & 592 \\
\hline $\begin{array}{l}\text { Sodium alendronate } \\
\text { and cancer }\end{array}$ & 1 & 6 & 4 & 73 & 3 & 80 & 7 & 1070 \\
\hline $\begin{array}{l}\text { Nanoparticle and } \\
\text { cancer }\end{array}$ & 10 & 20064 & 46795 & 7699 & 28.656 & 54167 & 12986 & 92000 \\
\hline
\end{tabular}

Fonte: Autores (2021).

Uma das áreas mais promissoras no uso de nanopartículas é a vetorização de medicamentos anticâncer (Massey et al., 2016; Kwon, et al., 2019), antibióticos (Pintoalphandary et al., 2000) e anti-inflamatórios não esteroidais (NSAIDS) (Rosado; Silva; Reis, 2013), principalmente por via parenteral, visando distribuí-los de forma mais seletiva, aumentando assim o índice terapêutico. Além disso, o alto custo de desenvolvimento de novas moléculas bioativas torna os sistemas nanoestruturados de fármacos uma das áreas mais promissoras da indústria farmacêutica. Isso porque novas formulações em nanopartículas podem contribuir com a farmacologia, visto que muitas drogas promissoras são descartadas devido a efeitos colaterais ou baixa disponibilidade. Atualmente, as empresas farmacêuticas têm investido em pesquisa e desenvolvimento de novos medicamentos nanotecnológicos, principalmente para liberação de medicamentos (Boni, et al., 2016). 
Figura 5 - Depósito de patentes relacionados a alendronato de sódio, nanopartículas e câncer nas principais plataformas de pesquisa tecnológica.

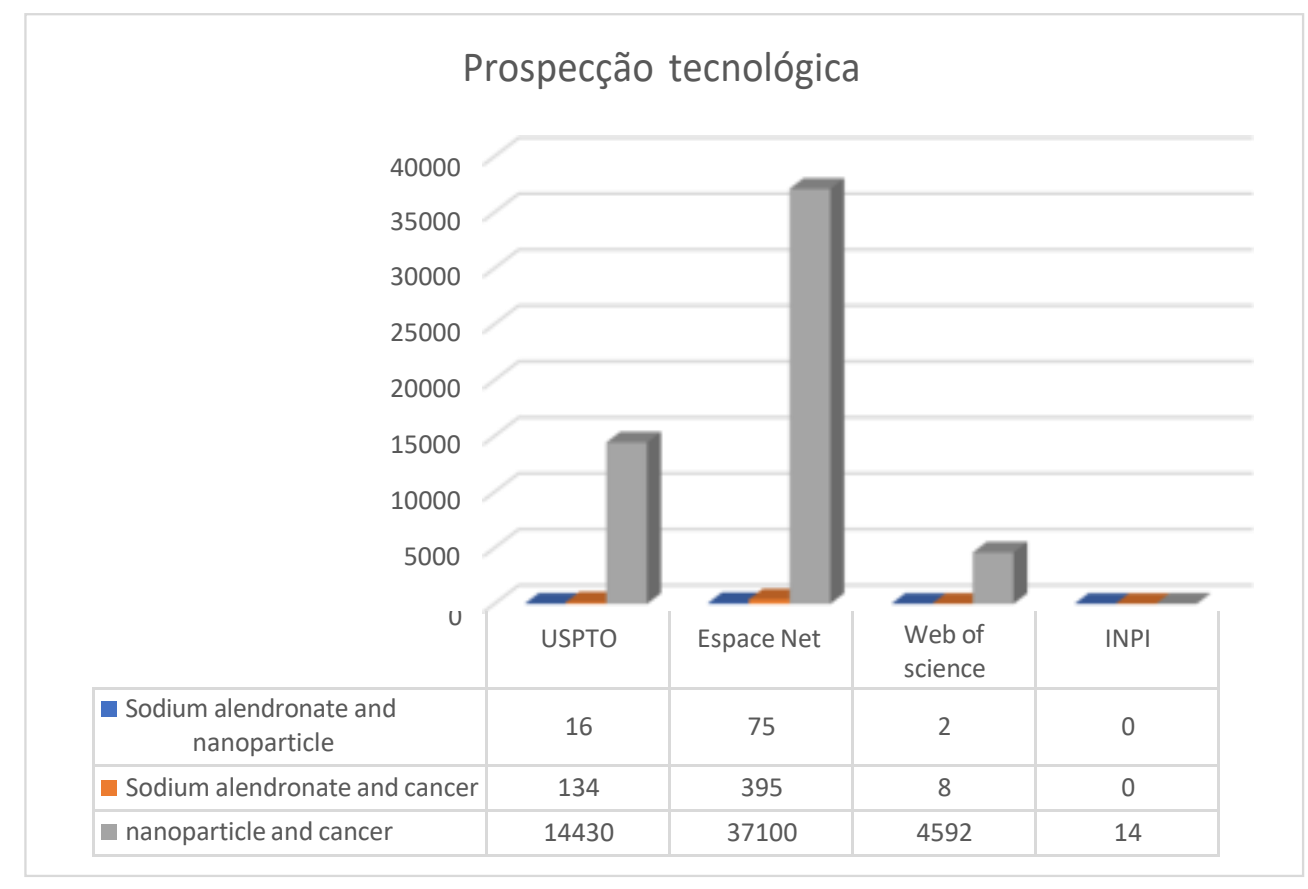

Fonte: Autores (2021).

Medicamentos podem ser encapsulados por diferentes mecanismos, incluindo solubilização ou retenção em nanopartículas, ligação ou adsorção. Em condições ideais, eles podem transportar medicamentos para destinos específicos para que seus efeitos terapêuticos possam ser realizados com segurança (Kato, 2019). Dentre os sistemas de nanocarreadores farmacológicos, destacam-se as nanopartículas poliméricas. Nanopartículas poliméricas representam uma escolha tecnológica com excelente biocompatibilidade, não imunogenicidade, baixa toxicidade e biodegradabilidade. Em comparação com outros sistemas carreadores de fármacos (como os lipossomas), uma das vantagens do uso de nanopartículas poliméricas é que, além de maior estabilidade e durabilidade, o custo de obtenção dos polímeros é menor. Estes podem existir na forma de nanocápsulas ou nanoesferas (Rai; Alwani; Badea, 2019).

Mesmo com essa possibilidade de junção de medicamentos e nanopartículas, muitas possibilidades precisam ser exploradas. Em se tratando da pesquisa relacionando o alendronato de sódio e nanopartículas, foram encontrados poucos dados, tanto para a prospecção científica quanto para a tecnológica. Ainda assim, foi possível verificar que as pesquisas com essa relação estão aumentando, mesmo que de forma lenta, isso fica evidente na figura 4, onde observa-se um aumento de de publicações contendo alendronato de sódio e nanopartículas, com um pequeno decréscimo no ano de 2020, possivelmente associado à pandemia da COVID-19. O mesmo déficit foi observado na prospecção tecnológica, onde os pedidos de patente relacionando ALD e nanopartículas foi considerável (máx. 75), entretanto como as buscas não são especificamente precisas, alguns trabalhos podem apresentar apenas as duas palavras citadas em tópicos importantes, mas sem correlacioná-los.

Esses dados sugerem que o potencial do ALD, ainda precisa ser melhor explorado, principalmente a sua associação com nanopartículas, que pode, eventualmente reduzir seus efeitos colaterais e potencializar sua atividade. Esse é um fármaco produzido por múltiplas indústrias, existindo no mercado brasileiro múltiplas especialidades farmacêuticas, podendo inclusive ser transformado em medicamentos refinados (Def, 2004). Pode ser administrado por via oral ou como um único comprimido de alendronato $(\mathrm{C} 4 \mathrm{H} 12 \mathrm{NNaO} 7 \mathrm{P} 2.3 \mathrm{H} 2 \mathrm{O})$ na forma de um tri-hidrato $(\mathrm{C} 4 \mathrm{H} 12 \mathrm{NNaO} 7 \mathrm{P} 2.3 \mathrm{H} 2 \mathrm{O})$ em doses de 5,10 e $70 \mathrm{mg}$ de alendronato (C4H13NO7P2) por comprimido. Além disso, o alendronato é um amino-bisfosfonato, semelhante ao pirofosfato, 
que é uma substância endógena que regula o metabolismo do cálcio. Possui um grupo amino em sua estrutura, que possui maior eficácia do que outras drogas de outras classes (Scarpa et al, 2010). Mesmo com os dados já apresentados, ainda se faz necessário mais investigações e associações com esse fármaco tão promissor, que além de apresentar possibilidade de nanoestruturação, possui também atividade anticâncer descrita na literatura.

Tratando-se do efeito anticâncer desse fármaco, um padrão semelhante a sua associação com nanopartículas foi observado. Um máximo de 1070 artigos foram encontrados na base do Google acadêmico, e mínimo de 1 artigo na base Scielo, para a busca por "sodium alendronate and cancer" (Figura 3 e tabela 1) e um aumento na produção de trabalhos científicos nos últimos 10 anos. Este fármaco ainda tem seu mecanismo de ação pouco elucidado, entretanto, alguns trabalhos já relatam que o mesmo causa apoptose, por vias de ativação de caspase-3, MAPK, JNK e p38 (Idris et al., 2008; Buranrat; Bootha, 2019). Essa atividade anticâncer, aparentemente está sendo bem explorada, pois na pesquisa tecnológica foram encontradas 395 patentes nesse âmbito, na base Espace Net, mas nenhuma na base nacional, o que mostra que esse a atividade anticâncer do ALD bem como suas melhorias por união com a nanotecnologia estão sendo melhores explorados em outros países, que não o Brasil.

\section{Conclusão}

Com o passar dos anos o câncer vem se tornando cada vez mais incidente, com um aumento constante no número de casos, consequentemente ocasionando um grande número de mortes. Nesse sentido, a busca por meios que facilitem, melhorem, aperfeiçoem e contribuam para o tratamento do câncer vem sendo crescente, e junto a isso o ramo da nanotecnologia também tem se desenvolvido bastante. As nanopartículas vêm sendo bastante trabalhadas e discutidas no meio científico, por terem capacidades muito bem aproveitadas pelos fármacos, podendo aumentar sua eficiência, diminuir sua toxicidade e aumentar a biodisponibilidade.

Dito isto, com base nos resultados obtidos na busca de patentes sobre o uso do alendronato no tratamento do câncer e sua associação com nanopartículas, apresentou-se um resultado mediano de patentes publicadas, observando-se um resultado semelhante, no que se refere a publicação de artigos.

Deste modo, fica evidenciado que o desenvolvimento de nanopartículas associadas ao alendronato de sódio é um campo ainda em desenvolvimento, especialmente quando direcionado ao tratamento do câncer. Logo, são necessários novos estudos que explorem essa associação, tendo em vista o potencial do alendronato de sódio e as vantagens associadas a nanopartículas, e sendo o câncer uma doença que carece do desenvolvimento de novas tecnologias direcionadas ao seu tratamento.

\section{Referências}

A Baudino, T. (2015). Targeted cancer therapy: the next generation of cancer treatment. Current drug discovery technologies, 12(1), 3-20. Abbas, Z., \& Rehman, S. (2018). An overview of cancer treatment modalities. Neoplasm, 1, 139-57.

Andreopoulou, E., Kelly, C. M., \& McDaid, H. M. (2017). Therapeutic advances and new directions for triple-negative breast cancer. Breast Care, 12(1), 2027.

Boni, F. I., Almeida, A., Lechanteur, A., Sarmento, B., Cury, B. S. F., \& Gremião, M. P. D. (2018). Mucoadhesive nanostructured polyelectrolytes complexes modulate the intestinal permeability of methotrexate. European Journal of Pharmaceutical Sciences, 111, 73-82.

Buranrat, B., \& Bootha, S. (2019). Antiproliferative and antimigratory activities of bisphosphonates in human breast cancer cell line MCF-7. Oncology letters, 18(2), 1246-1258.

Cataliotti, L., Buzdar, A. U., Noguchi, S., Bines, J., Takatsuka, Y., Petrakova, K., ... \& Tosello de Oliveira, C. (2006). Comparison of anastrozole versus tamoxifen as preoperative therapy in postmenopausal women with hormone receptor-positive breast cancer: The Pre-Operative "Arimidex" Compared to Tamoxifen (PROACT) trial. Cancer, 106(10), 2095-2103.

Chlebowski, R. T., Chen, Z., Cauley, J. A., Anderson, G., Rodabough, R. J., McTiernan, A., ... \& Wallace, R. B. (2010). Oral bisphosphonate use and breast cancer incidence in postmenopausal women. Journal of Clinical Oncology, 28(22), 3582. 
Coleman, R. E., \& McCloskey, E. V. (2011). Bisphosphonates in oncology. Bone, 49(1), 71-76.

DEF.(2004) Dicionário de Especialidades Farmacêuticas 2003/2004. Publicações Científicas. 32.ed. Rio de Janeiro:. p.88.

Dimer, F. A., Friedrich, R. B., Beck, R. C., Guterres, S. S., \& Pohlmann, A. R. (2013). Impactos da nanotecnologia na saúde: produção de medicamentos. Química Nova, 36, 1520-1526.

Idrees, H., Zaidi, S. Z. J., Sabir, A., Khan, R. U., Zhang, X., \& Hassan, S. U. (2020). A review of biodegradable natural polymer-based nanoparticles for drug delivery applications. Nanomaterials, $10(10), 1970$.

Idris, A. I., Rojas, J., Greig, I. R., van’t Hof, R. J., \& Ralston, S. H. (2008). Aminobisphosphonates cause osteoblast apoptosis and inhibit bone nodule formation in vitro. Calcified tissue international, 82(3), 191-201.

Iles, B., Ribeiro, I. N. S., Dourado, F. F., Ribeiro, F. D. O. S., de Araújo, A. R., de Oliveira, T. M., ... \& Medeiros, J. V. R. (2021). Alendronate sodiumpolymeric nanoparticles display low toxicity in gastric mucosal of rats and Ofcol II cells. NanoImpact, 100355.

Instituto Nacional de Câncer José Alencar Gomes da Silva (2019). Estimativa 2020: incidência de câncer no Brasil / Instituto Nacional de Câncer José Alencar Gomes da Silva. - Rio de Janeiro: INCA, 2019.

Kato, T. (2019). Encapsulated drugs in targeted cancer therapy. In Controlled drug delivery (pp. 189-240). CRC Press.

Kwon, S., Yoo, K. H., Sym, S. J., \& Khang, D. (2019). Mesenchymal stem cell therapy assisted by nanotechnology: a possible combinational treatment for brain tumor and central nerve regeneration. International journal of nanomedicine, 14, 5925.

Lipton, A. (2008). Emerging role of bisphosphonates in the clinic-antitumor activity and prevention of metastasis to bone. Cancer treatment reviews, 34, S25-S30.

Massey, A. S., Pentlavalli, S., Cunningham, R., McCrudden, C. M., McErlean, E. M., Redpath, P., ... \& McCarthy, H. O. (2016). Potentiating the anticancer properties of bisphosphonates by nanocomplexation with the cationic amphipathic peptide, RALA. Molecular pharmaceutics, 13(4), $1217-1228$.

MN Iqbal, H., MV Rodriguez, A., Khandia, R., Munjal, A., \& Dhama, K. (2016). Recent trends in nanotechnology-based drugs and formulations for targeted therapeutic delivery. Recent patents on inflammation \& allergy drug discovery, 10(2), 86-93.

Neville-Webbe, H. L., Gnant, M., \& Coleman, R. E. (2010, June). Potential anticancer properties of bisphosphonates. In Seminars in oncology (Vol. 37, pp. S53-S65). WB Saunders.

Oliveira, V. M. D., Aldrighi, J. M., \& Bagnoli, F. (2011). Evidências da ação dos bisfosfonatos na prevenção do câncer de mama. Revista da Associação Médica Brasileira, 57(1), 7-8.

Papamitsou, T., Sotiriou, S., Papakoulas, A., Toskas, A., Kamperis, D., Karachrysafi, S., ... \& Sioga, A. (2019). Alendronate effect in esophagus, stomach and liver: an animal based pathological study. Histology and histopathology, 35(4), 417-422.

Pinto-Alphandary, H., Andremont, A., \& Couvreur, P. (2000). Targeted delivery of antibiotics using liposomes and nanoparticles: research and applications. International journal of antimicrobial agents, 13(3), 155-168.

Rai, R., Alwani, S., \& Badea, I. (2019). Polymeric nanoparticles in gene therapy: New avenues of design and optimization for delivery applications. Polymers, 11(4), 745.

Rosado, C., Silva, C., \& Reis, C. P. (2013). Hydrocortisone-loaded poly ( $\varepsilon$-caprolactone) nanoparticles for atopic dermatitis treatment. Pharmaceutical development and technology, 18(3), 710-718.

Russell, R. G. G. (2011). Bisphosphonates: the first 40 years. Bone, 49(1), 2-19.

Russell, R. G. G., \& Rogers, M. J. (1999). Bisphosphonates: from the laboratory to the clinic and back again. Bone, 25(1), 97-106.

Scarpa, L. C., de Mello Leite, L. C., de Lacerda, J. C. T., \& Arantes, D. C. B. (2010). Osteonecrose nos ossos da maxila e mandíbula associada ao uso do bifosfonato de sódio. Revista Brasileira de Pesquisa em Saúde/Brazilian Journal of Health Research.

Schaffazick, S. R., Guterres, S. S., Freitas, L. D. L., \& Pohlmann, A. R. (2003). Caracterização e estabilidade físico-química de sistemas poliméricos nanoparticulados para administração de fármacos. Química nova, 26, 726-737.

Sousa, P. S. A., Nogueira, S. S., Ayala, K. N. R., Silva, P. C., Santos, E. S., Sa, R. E., Lima Neto, F. E. M., Lima, J. R. C., Rodrigues, K. A. F., Rocha, J. A., Veras, L. M. C. (2021). Prospecção científica e tecnológica de Pilocarpus microphyllus e do alcaloide epiisopiloturina com ênfase na atividade antileishmania. Research, Society And Development, v. 10, p. 1-17.

Steenbruggen, T. G., van Ramshorst, M. S., Kok, M., Linn, S. C., Smorenburg, C. H., \& Sonke, G. S. (2017). Neoadjuvant therapy for breast cancer: established concepts and emerging strategies. Drugs, 77(12), 1313-1336.

Van den Wyngaert, T., Claeys, T., Huizing, M. T., Vermorken, J. B., \& Fossion, E. (2009). Initial experience with conservative treatment in cancer patients with osteonecrosis of the jaw (ONJ) and predictors of outcome. Annals of Oncology, 20(2), 331-336.

Ye, F., Zhao, Y., El-Sayed, R., Muhammed, M., \& Hassan, M. (2018). Advances in nanotechnology for cancer biomarkers. Nano Today, $18,103-123$. 